\title{
Use cases to business modelling of satellite backhaul in $5 \mathrm{G}$ Simon Watts ${ }^{1}$, Konstantinos Liolis ${ }^{2}$, Salvador Diaz ${ }^{3}$, Marlies Van der Wee ${ }^{4}$
}

\author{
${ }^{1}$ AGS, Avanti Communications, Cobham House, 20 Black Friars Lane, London, UK \\ ${ }^{2}$ SES S.A, Château de Betzdorf, L-6815 Betzdorf, Luxembourg \\ ${ }^{3}$ British Telecommunications PLC, Adastral Park, Ipswich, UK \\ ${ }^{4}$ Ghent University - imec, Ghent, Belgium
}

\section{Keywords: SATCOM, 5G, EMBB, BACKHAUL, INTEGRATION}

\begin{abstract}
The H2020 project SaT5G [10] [13] is researching and developing the capability to seamlessly integrate satellite communication (satcom) backhaul links into $5 \mathrm{G}$ to support enhanced Mobile Broadband (eMBB). Four relevant use cases and a set of KPIs have been identified. A variety of different integration architectures are described. These factors have then been reviewed to allow business modelling for the new paradigms brought by $5 \mathrm{G}$, and the role of a bandwidth broker has been identified.
\end{abstract}

\section{Introduction}

The H2020 project "SaT5G" (Satellite and Terrestrial network for 5G) [10], [13] is developing the role of satcom in $5 \mathrm{G}$ by defining optimal satellite-based backhaul and traffic offloading solutions. The goal is to enhance $5 \mathrm{G}$ networks with the satcom benefits by a seamless integration and to create new business opportunities. To achieve such goals, SaT5G is researching, developing and validating the optimal satellite integration and creating new business roles in the early stages. The SaT5G project structure is shown in the following figure.

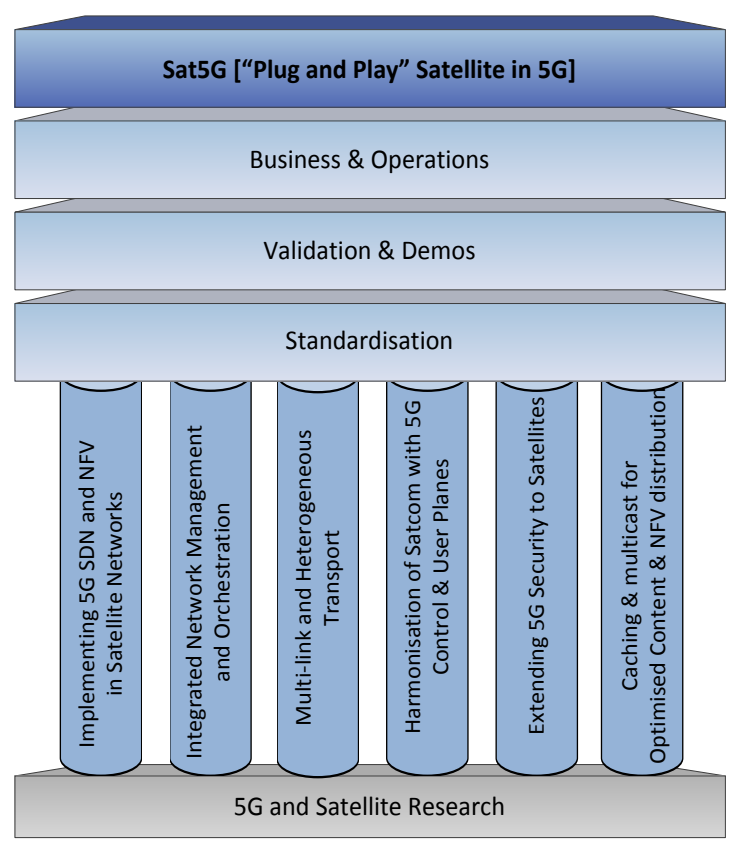

Figure 1: SaT5G Project Structure
Based on the anticipated market needs, SaT5G focuses on the eMBB Usage Scenario for 5G. SaT5G addresses the mobile network operators' (MNOs) needs for the inclusion of satellite in the early $5 \mathrm{G}$ roll out; bypassing congested backhaul and offloading high bandwidth video download have been found to be the major drivers. SaT5G is investigating novel business models and economically viable operational collaborations that integrate the satellite and terrestrial stakeholders in a win-win situation.

This paper addresses the arising business models, relating them to the project use cases, architecture and Key Performance Indicators (KPIs).

The remainder of this paper is organized as follows: section 2 provides an overview of the most promising and relevant satellite use cases in 5G investigated as part of the SaT5G project. Section 3 elaborates on the KPIs addressed by the SaT5G project and their mapping to the satellite use cases. Section 4 addresses the architecture options for satellite integration into $5 \mathrm{G}$, with focus on satellite backhaul architectures. Section 5 addresses the business modelling aspects; finally section 6 concludes the paper.

\section{Use Cases}

\subsection{Selected use cases}

By following the methodology described in [1] [2], SaT5G has selected four Satellite Use Cases for eMBB to focus its efforts on (see also figure 2 following):

1) Edge delivery and offload of multimedia content and MEC VNF software, through multicast and caching to optimise the operation and dimensioning of the $5 \mathrm{G}$ network infrastructure;

2) $5 G$ fixed backhaul, to provide $5 G$ service especially in areas where it is difficult or not possible to deploy terrestrial communications;

3) $5 G$ to premises, to provide $5 G$ service into home/office premises in underserved areas via hybrid terrestrialsatellite broadband connections;

4) $5 G$ moving platform backhaul, to support $5 \mathrm{G}$ service on board moving platforms, such as aircraft, vessels, trains, etc. 


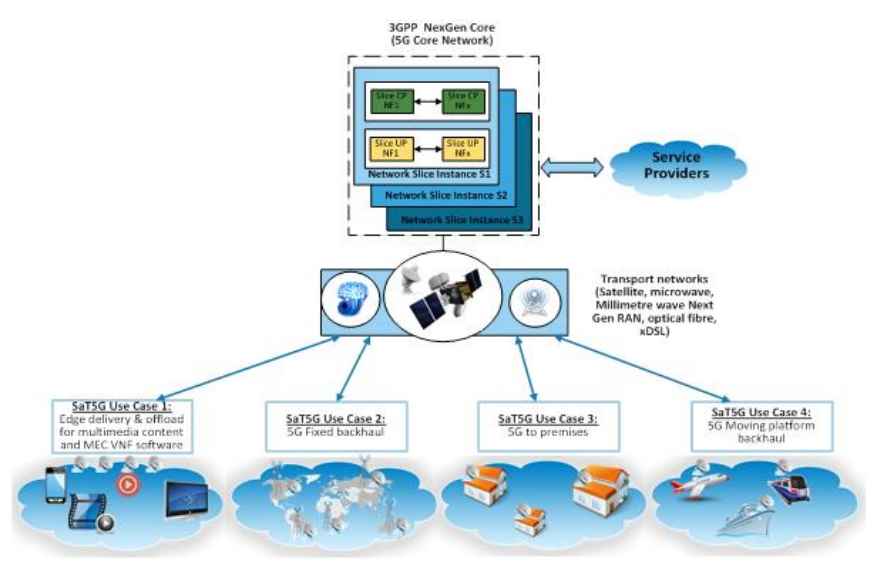

Figure 2: SaT5G Use Cases

Each use case has two or more specific scenarios as shown in table 1 below. Selected scenarios are used for the business analysis and will be used later in the project for the validation tests and demonstrations.

Table 1: SaT5G Use Cases and Scenarios

\begin{tabular}{|c|c|}
\hline SaT5G Use Cases & Scenarios for SaT5G Use Cases \\
\hline \multirow{2}{*}{$\begin{array}{l}\text { SaT5G Use Case 1: } \\
\text { Edge delivery \& } \\
\text { offload for } \\
\text { multimedia content } \\
\text { and MEC VNF s/w }\end{array}$} & $\begin{array}{l}\text { Scenario 1a: Offline multicasting and } \\
\text { caching of video content and VNF } \\
\text { software through satellite links }\end{array}$ \\
\hline & $\begin{array}{l}\text { Scenario 1b: Online prefetching of } \\
\text { video segments through satellite links }\end{array}$ \\
\hline \multirow{3}{*}{$\begin{array}{l}\text { SaT5G Use Case 2: } \\
\text { 5G Fixed backhaul }\end{array}$} & $\begin{array}{l}\text { Scenario 2a: Satellite backhaul to } \\
\text { groups of cell towers }\end{array}$ \\
\hline & $\begin{array}{l}\text { Scenario } 2 \mathrm{~b} \text { : Satellite backhaul to } \\
\text { individual cell towers }\end{array}$ \\
\hline & $\begin{array}{l}\text { Scenario 2c: Satellite backhaul to } \\
\text { individual small cells }\end{array}$ \\
\hline \multirow{2}{*}{$\begin{array}{l}\text { SaT5G Use Case } 3 \text { : } \\
\text { 5G to premises }\end{array}$} & $\begin{array}{l}\text { Scenario 3a: Hybrid Multiplay } \\
\text { (satellite/xDSL) at home/office } \\
\text { premises in underserved areas }\end{array}$ \\
\hline & $\begin{array}{l}\text { Scenario 3b: Hybrid Multiplay } \\
\text { (satellite/cellular) at home/office } \\
\text { premises in underserved areas }\end{array}$ \\
\hline \multirow{3}{*}{$\begin{array}{l}\text { SaT5G Use Case 4: } \\
\text { 5G Moving } \\
\text { platform backhaul }\end{array}$} & $\begin{array}{l}\text { Scenario 4a: Updating content for on- } \\
\text { board systems and grouped media } \\
\text { request by the moving platform } \\
\text { company }\end{array}$ \\
\hline & $\begin{array}{l}\text { Scenario 4b: Broadband access for } \\
\text { passengers and individual media } \\
\text { requests }\end{array}$ \\
\hline & $\begin{array}{l}\text { Scenario 4c: Business and technical } \\
\text { data transfer for the moving platform } \\
\text { company }\end{array}$ \\
\hline
\end{tabular}

Scenario $2 b$ is frequently used within SaT5G as the baseline description from which other scenarios are extrapolated. It is more fully defined as:

"Satellite backhaul to a single cell tower located in a rural area in the EU covering two villages about $5 \mathrm{~km}$ apart and a rural main road. The villages are home to 300 families, in summer months an additional 50 families may be in holiday accommodation. The road can occasionally be busy with holiday traffic but is usually quiet. The predominant traffic on the cell is eMBB but there is some [very low level] $m M T C$ traffic generated by agri-tech."

\subsection{Background developments}

Satellite systems are evolving, with greater capacity (in the order of Tbps speed) with accordingly lower cost per bit and better connectivity which makes the integration of satellite with terrestrial mobile networks a compelling commercial proposition. Future satellite constellations will complement this with lower latency connections.

The business aim is to improve customer experience and value, with lower incremental capital cost than fixed network upgrades. The near $100 \%$ coverage provided by satellite, and the characteristic distance-independent cost of adding users, is increasingly desirable for MNOs for capacity and coverage. Satcom integration within 5G will expand the opportunity for MNOs by enabling fast roll out of services in a way that has not been possible for $3 \mathrm{G} / 4 \mathrm{G}$.

\subsection{Related deployment opportunities}

The opportunities for satcom offering eMBB backhaul have been found to fall into two categories where satellites offer a provision of alternative or overlay paths for:

\subsubsection{Offloading and caching in the fixed network:}

(a) Connecting data-centres to 5G-core network gateways where there are no terrestrial or economically viable paths, and;

(b) Connecting the core network to semi-centralised or centralised virtualised gNB processing centres.

2.3.2 Backhaul: connections to 5G cells in all layers (macro, micro, etc).

Four main example opportunities have been identified:

(a) Backhaul to cells in remote areas that are installed to achieve a certain coverage obligation and where providing a terrestrial link is not economically viable;

(b) Cells that need fast deployment and cannot wait for terrestrial provisioning;

(c) Deployment of cells for temporary events; and

(d) Deployment of small mobile cells mounted on public transport, on high-end cars, or on emergency services vehicles. 
Satcom deployment can be valuable across multiple verticals; table 2 following illustrates this comparing a representative set of target verticals (building on both [2] and [11]) against the use cases.

Table 2: Relevance of SaT5G Use Cases to Market Verticals

\begin{tabular}{|c|c|c|c|c|c|c|c|}
\hline SaT5G Use Case & 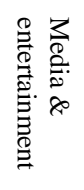 & 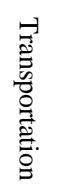 & 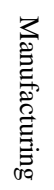 & 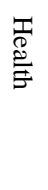 & 足: & 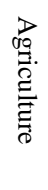 & 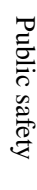 \\
\hline 1 - Edge delivery... & $\checkmark$ & $\checkmark$ & & & & & $\checkmark$ \\
\hline 2 - Fixed backhaul & $\checkmark$ & $\checkmark$ & $\checkmark$ & $\checkmark$ & $\checkmark$ & $\checkmark$ & $\checkmark$ \\
\hline $3-5 \mathrm{G}$ to premises & $\checkmark$ & & & & & & \\
\hline 4 - Moving platforms & $\checkmark$ & $\checkmark$ & & $\checkmark$ & $\checkmark$ & & $\checkmark$ \\
\hline
\end{tabular}

\section{Key performance indicators (KPIs) 3.1 The role of KPIs}

The main SaT5G goal is the seamless integration of satellite networks into 5G standard networks. The technical and the economic goals have different challenges which are addressed using the use cases previously defined. To be considered a $5 \mathrm{G}$ network, the satellite network requires a minimum set of capabilities. These capabilities are defined using the well-known approach, addressed in multiple standardization bodies like 3GPP, which defines the minimum requirements. These requirements take into account parameters like throughput, delay, reliability, etc. The KPIs are used to measure such requirements. To avoid any confusion between vendors, SaT5G describes how the KPIs are measured.

The definition of the KPIs is the starting point to solve the technical challenges when the next network generation is defined. In SaT5G, a subset of the defined KPIs is used by the business models to evaluate the cost of the solution. By considering the business models in these early stages, SaT5G is differentiated from previous attempts to introduce satellites into terrestrial cellular standards. The business models will guide the technical work during the process minimizing efforts on non-relevant work.

\subsection{Satellite and $5 G$ KPIs}

The list below shows the subset of satellite KPIs which are considered for business evaluation. Bandwidth is a limited resource that is shared between multiple operators and links. Satellite total throughput is another important parameter to be considered by the business models as it has a direct impact on the service that operators provide to their clients. These KPIs mixed with the coverage area, the traffic density and the satellite backhaul data rate are used to evaluate the cost per bit per user.

Spectrum usage: Usually specified in $\mathrm{GHz}$ defines the spectrum per beam.
Satellite bandwidth: defined in $\mathrm{MHz}$; representing the total allocable bandwidth or the power equivalent bandwidth depending on the service characteristics, the satellite network operator (SNO) procuring the necessary systems to terminate and manage the capacity. The same bandwidth is frequently reused by the use of multiple users and gateway beams.

Satellite throughput: in Mbps; the SNO using the provided systems to manage service levels to their user base. Required Mbps depends on the use case and the service.

Satellite backhaul data rate: The allocated data rate in Mbps for each link between the satellite gateway and remote satellite terminal connected to a gNB. This is usually less than the peak data rate per link.

Maximum satellite roundtrip delay: quoted in $\mathrm{ms}$ and varies per satellite orbit (GEO 600ms, MEO 150ms and LEO $30 \mathrm{~ms}$ making some allowance for signal processing delays).

Coverage area: The coverage provided by each satellite including number or beams and level of frequency reuse.

\subsection{Mapping of SaT5G use cases to 5G PPP KPIs}

The following table shows how the four SaT5G use cases are able to contribute to the often referred to 5G PPP KPIs [12]. Of particular note perhaps is the ability of satcom to reduce end user perceived latency by placing key content in edge caches. Indeed, according to [5] and [6], paradoxically, the low latency requirement for $5 \mathrm{G}$ networks is a big ally for satcom as many new locations for content servers will be required. In the transition to $5 \mathrm{G}$, content needs to be moved to the edge, densifying CDN networks and making satellite multicast a viable option. In this context, satcom can indeed help $5 \mathrm{G}$ networks to achieve low latency by multicasting content to caches located at individual cells, even in places without fibre.

Table 3: Mapping of SaT5G use cases to 5G PPP KPIs

\begin{tabular}{|c|c|c|c|c|c|}
\hline SaT5G Use Case & 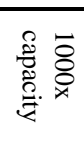 & 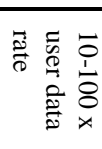 & 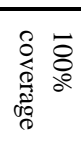 & 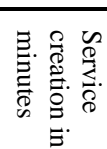 & $\begin{array}{l}\hat{B} \\
\vec{\Xi} \\
\vec{\Xi}\end{array}$ \\
\hline $1-$ Edge delivery... & $\checkmark$ & $\checkmark$ & $\checkmark$ & $\checkmark$ & $\checkmark$ \\
\hline 2 - Fixed backhaul & $\checkmark$ & $\checkmark$ & $\checkmark$ & $\checkmark$ & \\
\hline $3-5 \mathrm{G}$ to premises & $\checkmark$ & $\checkmark$ & $\checkmark$ & $\checkmark$ & $\checkmark$ \\
\hline 4 - Moving platforms & $\checkmark$ & $\checkmark$ & $\checkmark$ & $\checkmark$ & $\checkmark$ \\
\hline
\end{tabular}

\section{Architecture}

4.1 Roadmap and technical requirements for satellite architectures

One key part of the SaT5G project has been to look at today's satellite network architectures and compare these with the emerging 3GPP architectures for 5G networks looking at issues such as control and user plane functions, network slicing, virtualisation and end-to-enend service orchestration. 
A roadmap of different architectures showing increasing levels of $5 \mathrm{G}$ network integration to support non-terrestrial networks has been derived. This also addresses the four SaT5G use cases; looking at architectures for backhaul to fixed nodes and mobile platforms, for caching and multicast overlays, the end-to-end service delivery and defining the reference interface between satellite and terrestrial networks. These options are shown in the following figure.

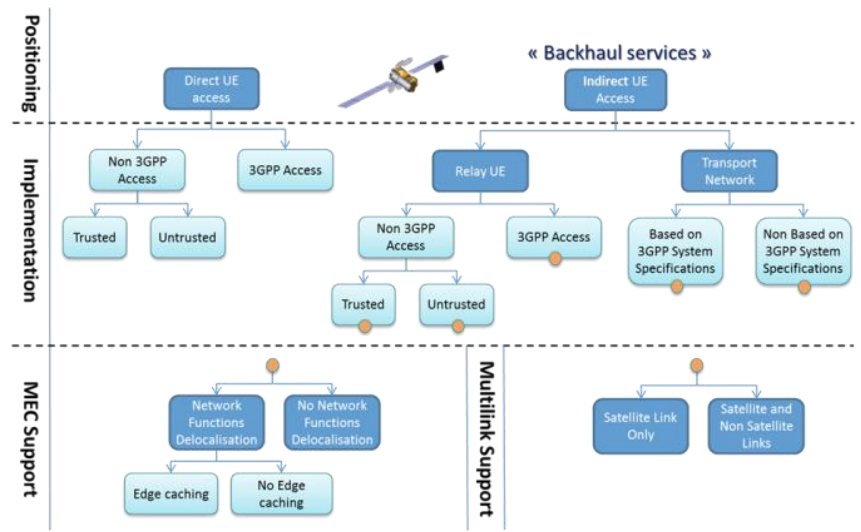

Figure 3: Options for satellite integration into $5 \mathrm{G}$

Several areas of integration have been identified like QoS adaptation, mobility management, beam management or multi-satellite connectivity. Below figure provides an overview about QoS adaptation of the user plane traffic between the terrestrial and the satellite link.

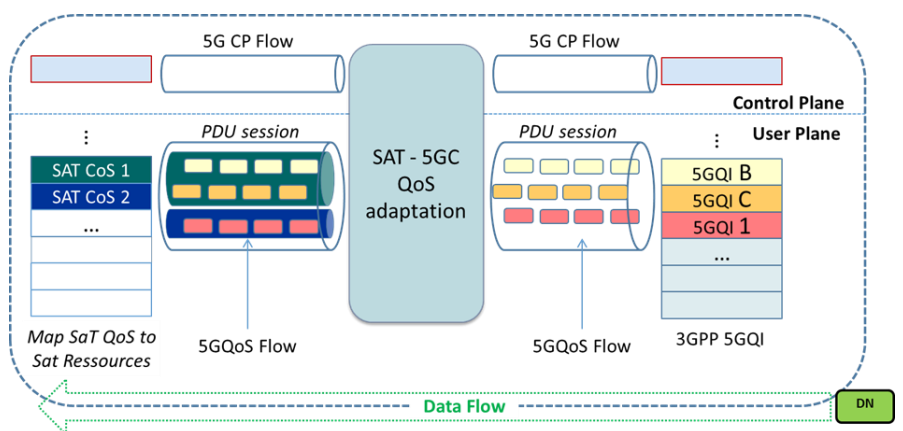

Figure 4: QoS adaptation between terrestrial and satcom 5G

\subsection{Backhaul architectures and 3GPP}

The SaT5G project has made major contributions to an ETSI report [4] and 3GPP study [9] that describes what it refers to as the non-terrestrial network (NTN) architectures in 3GPP compliant terminology. In addition, the report details the 3GPP interfaces presented to and across the satellite backhaul links. It documents the five scenarios as:

- Scenario A1 - Direct 3GPP access: The NTN enabled UE accesses the 5G Core Network via a 3GPP defined NTN NR-Radio Access. UE management applies to the NTN enabled UE.
- $\quad$ Scenario A2 - Indirect 3GPP access: 5G UE are served by an access point. This access point is served by a $3 \mathrm{GPP}$ defined NTN NR-Radio Access. UE management applies to the NTN terminal which is still named "NTN enabled UE". The NTN enabled UE endorses a multiplexer node role.

- $\quad$ Scenario A3 - Indirect mixed 3GPP NTN access: 5G UE are served by an access point. This access point is served by a trusted mixed 3GPP NTN access network (see "Non-3GPP access network" definition). UE management applies to the NTN terminal which is named "NTN enabled UE". The NTN enabled UE endorses a multiplexer node role. Another wording for scenario A3 could be "Indirect 3GPP NTN access with non-3GPP L2, non-3GPP L1".

- Scenario A4 - Indirect access via transparent transport network: 5G UE are served by an access point. This access point is connected to the $\mathrm{CN}$ via a NTN transport network. The UE management does not apply to the NTN terminal. The NTN terminal may endorse a multiplexer node role. This NTN transport network is considered as trusted by the operator of the UEs.

- Scenario A5 - Indirect untrusted access: 5G UE are served by an access point. This access point is served by an untrusted non-3GPP or a mixed 3GPP NTN access network. UE management does not apply to the NTN terminal. The NTN terminal endorses a multiplexer node role.

One example showing the mapping of the 5G system reference points on a logical network representation is given in the following figure.

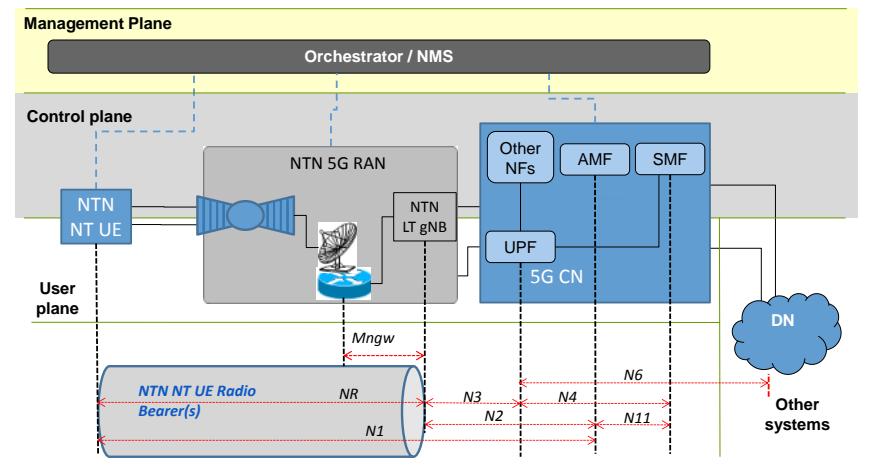

Figure 5: Mapping 5G system reference points

\subsection{Business implications on the architecture}

The business analysis is a SaT5G differentiator taking into account the implications of the architectures that vary depending on use case and operator deployment models. As such a series of business roles have been identified aligning with key architectural blocks. These then allow different value network configurations (interactions between stakeholders taking up these business roles) to be considered where the business roles can be assigned to different stakeholders in the use case specific value network. 
In reviewing the business implications of integrating satellite links in to future $5 \mathrm{G}$ networks, where there may be multiple satellite network operators (SNOs) and multiple terrestrial MNOs (the two stakeholders identified as core roles), we have identified the potential for a trusted third party to provide a brokerage service to provide the capacity where needed under well-defined contractual and commercial terms.

In the focus on the core roles, different business models can be formed based on three parameters:

- The number of SNOs, can be 1 or multiple (M) or served by a broker (B);

- How the satellite capacity is offered: fixed (F) or dynamic (D) resource allocation;

- The number of MNOs, can be 1 or multiple (M) or served by a broker (B).

For each of the use cases defined in SaT5G, the short term (reference column in the table) and the long term (most likely) combinations have been identified in the table below.

\begin{tabular}{lll}
\hline Use case & Reference & Most likely \\
\hline $\begin{array}{l}\text { Use case 1: Offline } \\
\text { multicasting and caching of } \\
\text { video content and VNF } \\
\text { software through satellite } \\
\text { links }\end{array}$ & $1-\mathrm{F}-1$ & $\begin{array}{l}\text { (1-F-M)_B or } \\
\text { 1-D-M }\end{array}$ \\
$\begin{array}{l}\text { Use case 2: Satellite } \\
\text { backhaul to individual cell } \\
\text { towers }\end{array}$ & $1-\mathrm{F}-1$ & (1-F-M)_B or \\
$\begin{array}{l}\text { Use case 3: Hybrid } \\
\text { Multiplay (satellite/xDSL) } \\
\text { at home/office premises in } \\
\text { underserved areas }\end{array}$ & $1-\mathrm{F}-1$ & $1-\mathrm{D}-\mathrm{M}$ \\
$\begin{array}{l}\text { Use case 4: Broadband } \\
\text { access for passengers and } \\
\text { individual media requests }\end{array}$ & $\begin{array}{l}\text { 1-F-M } \\
\text { (train), } \\
\text { (plane) }\end{array}$ & $\begin{array}{l}\text { M-D-M or } \\
\text { (M-D-M)_B }\end{array}$ \\
\hline
\end{tabular}

The broker role is shown in the following figure. The broker provides a way to share the satellite use risk between the SNOs and MNOs in a reasonably equitable fashion. Further analysis is ongoing on how this role might cater for both point-to-point traffic and multicast content delivery traffic.

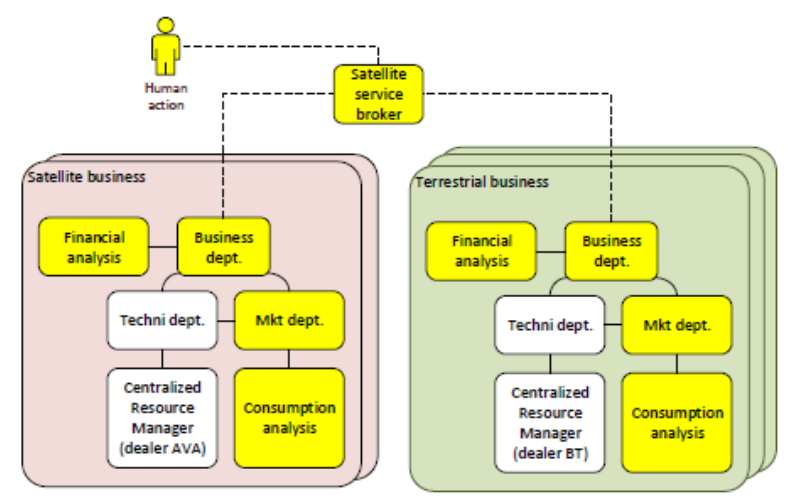

Figure 6: Role of Broker in Satellite Integration into 5G
It has also been identified that there is a strong correlation between the size of the request and the type of interaction required by the broker. An automatic process can provide near real time access to small well-defined capacity requirements while when the bandwidth requests and/or complexity increase, the need for manual intervention increases along with the notice period. This is illustrated in figure 7 below.

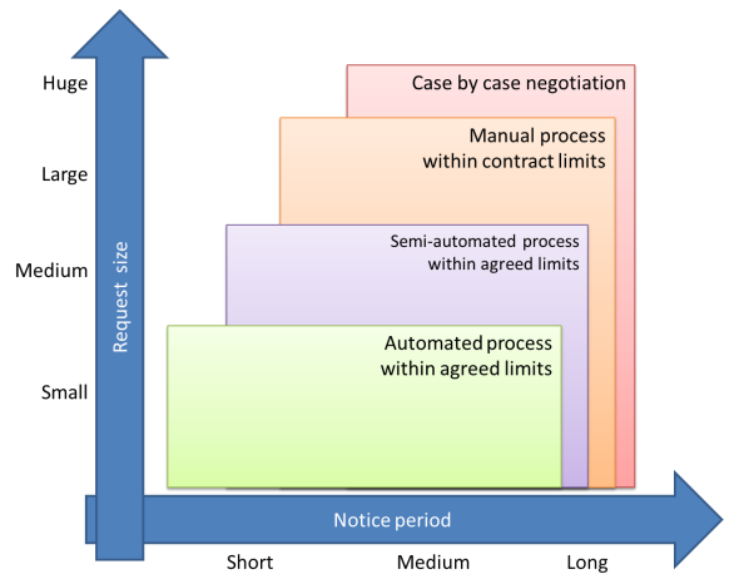

Figure 7: Request size, automation and notice period

\section{Business case \\ 5.1 Methodology}

Building on the KPI requirements, the SaT5G project has evaluated the economic and business impact by performing a techno-economic analysis of the use cases identified previously. Both an overall view on the value network (indicating the different roles needed for service delivery, as well as their interactions, [3]) and a focus on the core roles of satellite and mobile network operations (see figure below) are needed in order to decide on the viability of satellite integration into $5 \mathrm{G}$.

A model has been created that calculates the total cost of ownership (TCO) as well as the average revenue per user (ARPU) for the studied scenarios. The following results show the analysis for the specific case of the scenario $2 b$ (see section 2.1).

\subsection{Model and key assumptions}

The cost model develops a TCO for deploying the integrated terrestrial-satellite network, and consists of 4 parts (see figure 8 following):

- Cost model of the edge

- Cost model of the satellite network (for backhaul)

- Cost model for the $5 \mathrm{G}$ core network

- Overhead costs. 


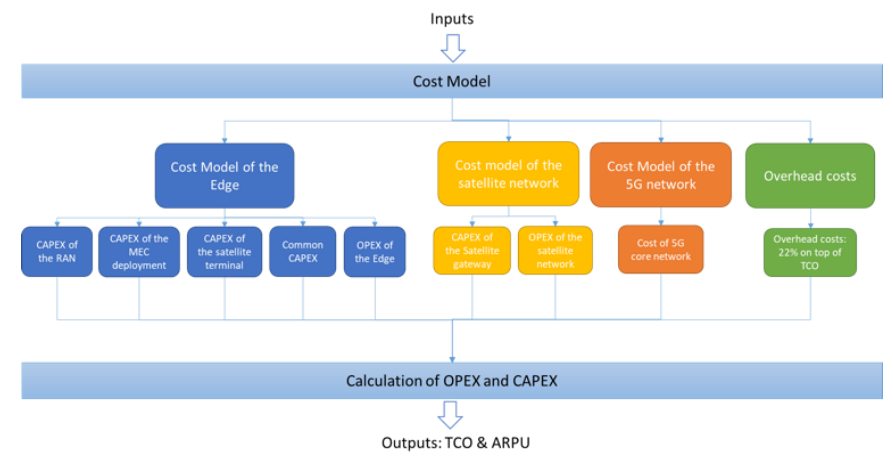

Figure 8: Cost model elements

The model calculates cost and the ARPU contribution needed based on a number of key assumptions:

- Hardware installation cost is $15 \%$ of the hardware costs [15];

- Maintenance cost is $10 \%$ of the CAPEX costs [8];

- Overhead cost is $22 \%$ on top of TCO [8];

- Project horizon is 5 years;

- The average revenue per user (ARPU) is the average cost per user (ACPU) plus a profit margin of $11 \%$ [14];

- We calculate a bit rate of between 53Mbps and 210Mbps per backhaul link;

- Finally, $80 \%$ of popular content is assumed to be cached.

The lower calculated bit rate assumes all 1050 end users consuming 6.5GB per month (based on the Cisco VNI report [16]) over the $5 \mathrm{G}$ backhaul; i.e. not via other routes such as $\mathrm{Wi}-\mathrm{Fi}$ as defined in scenario $2 \mathrm{~b}$ and represents a typical figure for Western-Europe in 2021. The higher figure represents 350 homes each consuming an average of $2 \mathrm{Mbps}$ (e.g. [7] states $1 \mathrm{Mbps}$ to $2 \mathrm{Mbps}$ for Western-Europe in 2020) during the peaks busy hour which is representative of the average traffic in Western-Europe for the whole home - in other words all their Internet capacity is provided by the $5 \mathrm{G}$ service and carried over the satellite backhaul.

The bandwidth demands will vary across the world; it is worth noting that the demand across Africa is forecasted to be around 1.5GB per UE per month [16] so the aggregated demand would be in the region of $12 \mathrm{Mbps}$ to $50 \mathrm{Mbps}$ and the modelled costs commensurately lower. In rural under developed regions such as those seen in Sub-Saharan Africa where cheap smartphones inevitably prevail, the backhaul traffic per link needs may well be below 10Mbps.

The calculated costs are presented as an ARPU contribution per end user to allow these to be readily compared with typical subscriber service charges. The calculated ARPU contributions exclude local end user taxation such as VAT.

\subsection{Findings}

Work is ongoing in SaT5G on refining the model and input assumptions, the model may also be amended as the detailed technical prototyping and validation tests are performed.
To date, the model shows that an ARPU contribution of between $€ 36$ and $€ 107$ (per month) in Western-Europe is calculated for SaT5G scenario $2 \mathrm{~b}$ where caching is not applied, whereas the scenario where $80 \%$ of popular content is cached at the edge, results in a reduction of the monthly ARPU to between $€ 9$ and $€ 28$. The reduction is mainly caused by a reduction in the needed throughput on the satellite backhaul link, leading to a reduction in operational expenses of around $75 \%$. In the real world an ARPU contribution between these two figures might be expected. These values indicate that whilst satcom backhaul ARPU contributions are too high for a total network deployment serving SaT5G use case 2 "5G satellite backhaul" needs; however the contribution are not astronomical and therefore reasonable for a few percent of sites.

The ARPU contributions for other regions will vary, for example in regions of lower demand such as Africa the ARPU contribution required will be much lower and therefore wider use will be possible.

Other use cases such as backhaul to mobile platforms will have different user bandwidth demands and satellite capacity costs due to different equipment requirements. The analysis against the other use cases has not been completed however by inspection use case 3 will be somewhat similar to the higher end ARPU contributions calculated for SaT5G scenario $2 b$.

\section{Conclusion}

A representative variety of four use cases and ten scenarios where the provision of $5 \mathrm{G}$ satellite backhaul services is likely to be of significant interest to both MNOs and SNOs has been presented. A mapping between the 5G KPIs and 5G satellite backhauls has been analysed. The architecture options for integrated $5 \mathrm{G}$ satellite backhaul delivery have been identified and inputs provided to 3GPP and ETSI.

From this work an initial business analysis has been undertaken looking at how the value matrices related to the different use cases. A cost model has been created that calculates the ARPU contributions needed per end user. A range of $€ 9$ to $€ 107$ per month per end user has been calculated for predicted Western-Europe traffic in 2021; depending on the amount of edge caching and whether this is only traffic to the handsets or to all devices in the home. This depends on how the real use cases align with those defined in SaT5G and described above.

These values indicate that whilst satcom backhaul ARPU contributions are too high for a total network deployment serving SaT5G scenario 2b "5G satellite backhaul" needs; the ARPU contribution are not excessive and therefore reasonable for a few percent of sites in Western-Europe.

Other regions and scenarios are the subject for further analysis within SaT5G and beyond. 


\section{Acknowledgements}

The SaT5G project has received funding from the European Union's Horizon 2020 research and innovation programme under grant agreement No 761413 [10], [13].

\section{References}

\section{Journal articles}

[1] K. Liolis, A. Geurtz, R. Sperber, D. Schulz, S. Watts, G. Poziopoulou, B. Evans, N. Wang, O. Vidal, B. Tiomela Jou, M. Fitch, S. Sendra Diaz, P. Sayyad Khodashenas, N. Chuberre, "Use Cases and Scenarios of 5G Integrated Satellite-Terrestrial Networks for Enhanced Mobile Broadband: The SaT5G Approach", Wiley's International Journal of Satellite Communications and Networking Special Issue "Integrated Satellite-Terrestrial Networks in Future Wireless Systems, 2018;1-22. https://doi.org/10.1002/sat.1245.

\section{Conference Papers}

\section{Book, book chapter and manuals}

[2] K. Liolis, A. Geurtz, R. Sperber, D. Schulz, S. Watts, G. Poziopoulou, B. Evans, N. Wang, O. Vidal, B. Tiomela Jou, M. Fitch, S. Sendra Diaz, P. Sayyad Khodashenas, N. Chuberre, "Satellite Use Cases and Scenarios for 5G eMBB", Book: "Satellite Communications in the 5G Era", Editors: S. Krishna Sharma, S. Chatzinotas, P.-D.M. Arapoglou, IET, 2018, ISBN: 978-1-78561-427-9.

[3] Verna Allee. "Value Network Analysis and Value Conversion of Tangible and Intangible Assets." Journal of Intellectual Capital. Publisher: Emerald Insights, Year: 2008, Volume: 9, Issue: 1, Page: 5 - 24

\section{Patents}

\section{Reports}

[4] ETSI TR 103611 "Integration scenarios of satellite and/or HAPS (High Altitude Platform Station) systems into 5G system and related architecture options", Draft ETSI Report Reference: SESSCN(18)000019r3 available online [5] GSMA Intelligence, "Understanding 5G," December 2014. [Online]. Available: https://www.gsmaintelligence.com/research/?file=1412085g.pdf\&download.

[6] NSR, "Wireless Backhaul via Satellite, 11th Edition," March 2017. [Online]. Available:

http://www.nsr.com/research-reports/satellite-

communications-1/wireless-backhaul-via-satellite-11thedition/.

[7] EC FP7 Project BATS, deliverable D5.2 "Cost Benefit Analysis", section 5.1.8, 29 April 2014

\section{Theses}

[8] Koen Casier (2010), Techno-economics of FTTH deployment in the presence of competition (Doctoral dissertation). Retrieved from

http://www.technoeconomics.ugent.be/output/phd_koen.pdf

\section{Standards}

[9] 3GPP TR 38.811 "Study on New Radio (NR) to support non-terrestrial networks", available online https://portal.3gpp.org/desktopmodules/Specifications/Specifi cationDetails.aspx? specificationId $=3$

\section{Websites}

[10] SaT5G (Satellite and Terrestrial network for 5G) project home page, http://sat5g-project.eu/, accessed 1 August 2018 [11] 5G: Serving Vertical Industries, 5G PPP, https://5g-ppp.eu/2nd-5g-vertical-workshop/, accessed 1

August 2018

[12] 5G PPP Key Performance Indicators, https://5g-ppp.eu/kpis/, accessed 1 August 2018

[13] EC CORDIS summary page for SaT5G, https://cordis.europa.eu/project/rcn/211060_en.html, accessed 1 August 2018

[14] A Fitness Program for GCC Telcos, A.T. Kearney, 2012 https://www.atkearney.com/documents/20152/434204/Telco_ Fitness.pdf/427dcc76-15f9-b9a3-0fd5-5c2e157918d6

[15] What is the average profit margin for a company in the telecommunications sector?, Investopedia retrieved 2018 , https://www.investopedia.com/ask/answers/060215/whataverage-profit-margin-company-telecommunicationssector.asp

[16] VNI Forecast Highlights Tool, Cisco, retrieved 2018, https://www.cisco.com/c/m/en_us/solutions/serviceprovider/vni-forecast-highlights.html\# 